\title{
Atomic Electron Correlations in Intense Laser Fields
}

\author{
L.F. DiMauro \\ B. Sheehy \\ B. Walker \\ P.A. Agostini \\ K.C. Kulander \\ This paper was prepared for submittal to the \\ 16th International Conference on Atomic Physics \\ Windsor, Ontario, Canada \\ August 2ळ7, 1998
}

September 3, 1998

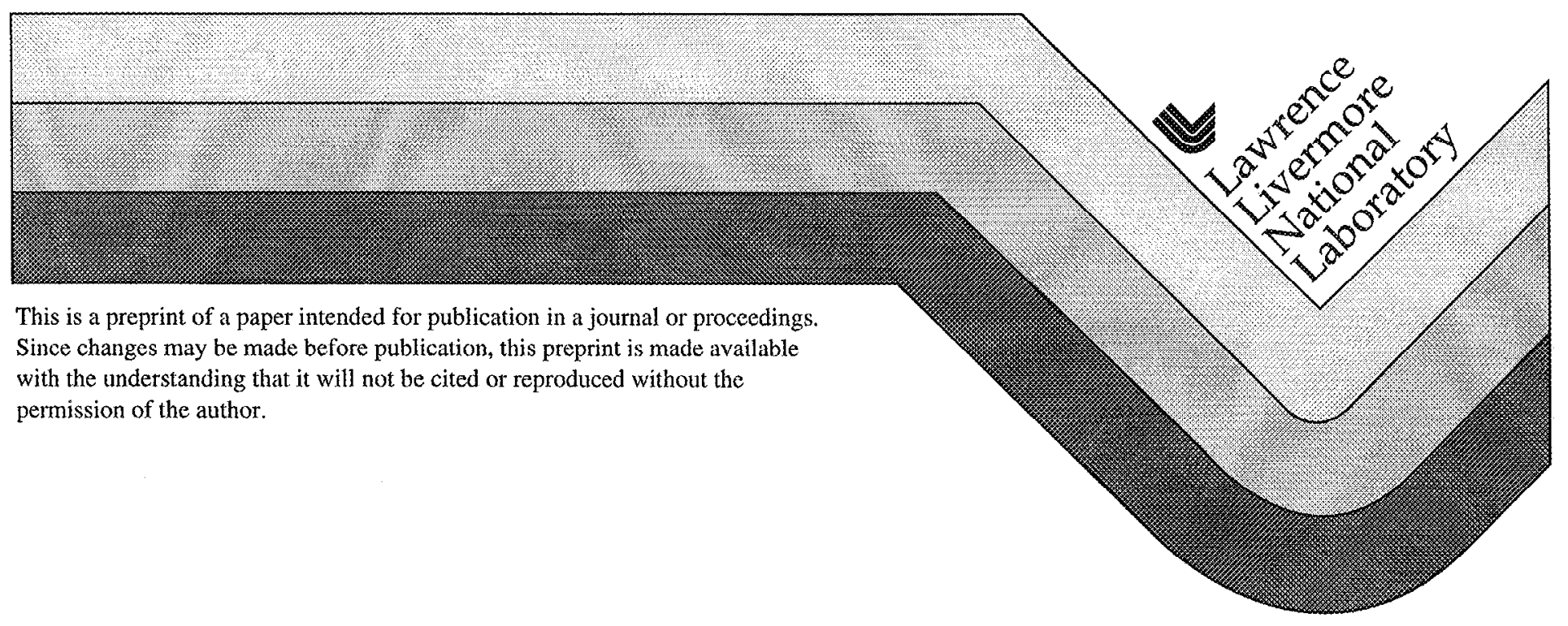




\section{DISCLAIMER}

This document was prepared as an account of work sponsored by an agency of the United States Govemment. Neither the United States Government nor the University of California nor any of their employees, makes any warranty, express or implied, or assumes any legal liability or responsibility for the accuracy, completeness, or usefulness of any information, apparatus, product, or process disclosed, or represents that its use would not infringe privately owned rights. Reference herein to any specific commercial product, process, or service by trade name, trademark, manufacturer, or otherwise, does not necessarily constitute or imply its endorsement, recommendation, or favoring by the United States Government or the University of Califomia. The views and opinions of authors expressed herein do not necessarily state or reflect those of the United States Government or the University of California, and shall not be used for advertising or product endorsement purposes. 


\title{
Atomic Electron Correlations in Intense Laser Fields
}

\author{
L. F. DiMauro*, B. Sheehy*, B. Walker*, P. A. Agostini ${ }^{\dagger}$ \\ and IK. C. Kulander. \\ "Brookhaven National Laboratory, Upton, NY 11979 \\ 'SPAM, Centre d'Etudes de Saclay, 91191 Gif Sur Yvette, France \\ † TAMP, Lawrence Livermore National Laboratory, Livermore, CA 94551
}

\begin{abstract}
This talk examines two distinct cases in strong optical fields where electron correlation plays an important role in the dynamics. In the first example, strong coupling in a two-electron-like system is manifested as an intensity-dependent splitting in the ionized electron energy distribution. This two-electron phenomenon (dubbed continuum-continuum Autler-Townes effect) is analogous to a strongly coupled twolevel, one-electron atom but raises some intriguing questions regarding the exact nature of electron-electron correlation. The second case examines the evidence for two-electron ionization in the strong-field tunneling limit. Although our ability to describe the oneelectron dynamics has obtained a quantitative level of understanding, a description of the two (multiple) electron ionization remains unclear.
\end{abstract}

\section{INTRODUCTION}

Many-body effects form the basis of a problem which is fundamental and central to our understanding of physics. In atomic physics, electronic correlation has been shown to play a prominent role both in the atomic structure and dynamics $[1,2]$. The study of multielectron atoms in intense laser fields raises similar issues concerning the influence of correlation in multiphoton excitation and ionization. As early as the mid-70s, correlation was considered an important element in explaining the anomalous multiple charge state distributions observed in the multiphoton ionization of allaline earth atoms [3]. Although this assignment in the end was in error, twenty years of ensuing intense field investigations have produced only a few unambiguous cases where correlation is relevant. The reader is referred to a recent comprehensive review of two electron atoms in intense fields by Lambropoulos et al. [4].

In this paper, we present two intense field experimental studies were the role of electron-electron correlation is important. The first scenario deals with the two-photon ionization of calcium [5], a two-electron like atom, with intense femtosecond light that is "resonant" with a core transition. The fundamental issue 
is what influence does a strongly driven core excitation have on the outgoing (ionized) photoelectron? In a second case, nonresonant ionization of helium by low energy $(1.6 \mathrm{eV})$ photons results in the anomalous double ionization yield [6] similar in character to that first observed in alkaline earth atoms by Suran and Zapescohyni [3]. However, unlike previous double ionization studies, helium ionization occurs in the strong field limit where the dynamics are dominated by tunneling. Even though much of the ambiguity present in the interpretation of a multiphoton ionization experiment is absent in the tunneling regime, the mechanism responsible for strong-field double ionization remains unclear. One significant difference between the two cases considered here is the external field strength as compared to the field between the valence clcctron and the core. In the first case, the laser field strength is $10^{-3}$ a.u. (perturbative) while for helium the amplitude is approaching 1 a.u. $(50 \mathrm{~V} / \mathrm{A})$ resulting in nonperturbative behavior. Thus in the language of Keldysh [7], calcium ionization occurs in the multiphoton limit $(\gamma>1)$ with strongly-coupled levels while helium tunnel ionizes $(\gamma<1)$. The Keldysh adiabaticity parameter, $\gamma$, is defined as the ratio of the laser frequency to the tunneling rate.

\section{Strongly Coupled Two-Electron Atom: The Continuum-Continuum Autler-Townes Effect}

Two bound states strongly coupled by an ac-field manifest an energy splitting which is due to the oscillation of population between the states in the presence of the driving field. This phenomenon, well known as the Autler-Townes doublet [8] when probed by a transition to a third level, or the Mollow triplet [9] when probed by resonance fuorescence, is usually not observed between a single bound state coupled to the continuum or between two coupled continua. In general, there is no population oscillation, as the breadth of the accessible phase space over which the coupling strength is distributed in the continuum makes excitation out of the initial state essentially irreversible. Thus, the initial state decays exponentially; saturation is reached without any splitting. For example, in multiphoton ionization, as the coupling between ionization continua is increased, only a broader distribution of the photoelectrons among those continua, separated by the photon energy, is achieved [above-threshold ionization (ATI)], with no splitting.

The situation is different for a two-electron atom where coupling between continua can produce a final state splitting if the driving laser field is resonant with some ionic core transition [10-13]. Physically, the reason for this is that the electron-electron interaction transfers the energy shift of the core electron to the outgoing electron. This has been dubbed "coherence transfer" by Ref.-110].

One simple way to understand this effect is to think of the final ionic state as split by the resonant (core)-interaction, thus the outgoing electron sees two asymptotic energy limits separated by the Rabi frequency $\Omega=\mu_{ \pm} \mathcal{E} / \hbar$, where $\mu_{ \pm}$is the ionic dipole and $\mathcal{E}$ the electric field. To emphasize the fact that it is 


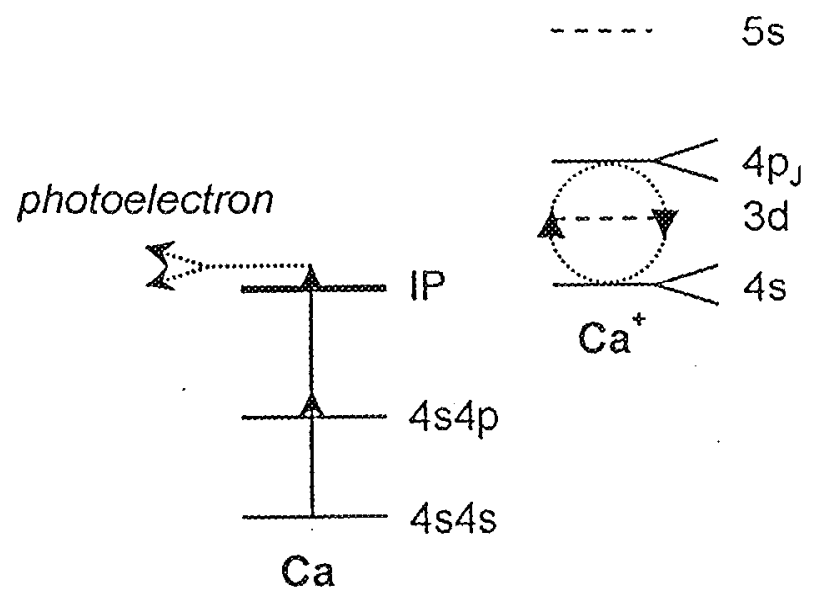

FIGURE 1. Simplified level diagram of calcium showing the 2-photon ionization of the neutral and the $4 s \Leftrightarrow 4 p_{J}$ coupled (split) core transition. The splitting of the photoelectron energy due to the continuum-continuum coupling is illustrated by the dotted line.

actually two continua that are coupled, one can talk about continuum-continuum Autler-Townes splitting. Dynamically, the outer electron is being ionized and, at the same time, the core-electron is driven in a Rabi oscillation. Note that the splitting would be reflected in Rydberg states as well [11]. Actually, the timeevolution of a Rydberg wave-packet under strong coupling of the core-electron gives rise to very interesting effects as discussed by Hanson and Lambropoulos [15]. For the phenomenology of strong-field optical resonance in two-level systems, the reader is referred to the literature [16]. We just summarize the general behavior of the photoelectron energy spectrum "on" resonance. At low intensity, the spectrum would consist of a single energy peak, as the intensity increases the peak will be symmetrically split by an amount proportional to the square root of the intensity. One should also recall that "on" resonance, the states are actually a linear superposition of bare states, thus any labeling of the split components by bare state quantum numbers is arbitrary.

The experimental realization [5] of this phenomenon uses a two-photon ionization scheme, as illustrated in Fig. 1. The initial bound statc is the calcium ground state $4 \mathrm{~s}^{2}{ }^{1} \mathrm{~S}_{0}$, and the first and second continua are the $|4 s, \epsilon\rangle$ and $\left|4 p_{J}, \epsilon\right\rangle$, respectively. Grobe and Eberly [10] showed that these are the minimum ingredients necessary to generate the effect. As a first approximation, the continuum-continuum coupling can be estimated by using the known bound $4 s \rightarrow 4 p$ transition strength in $\mathrm{Ca}^{+}$. The dipole strength is approximately 1.5 atomic units (a.u.), which for a moderate field strength of $3 \times 10^{-3}$ a.u. (intensity equal to $300 \mathrm{GW} / \mathrm{cm}^{2}$ ) yields an easily observable Rabi splitting $(\Omega)$ of about $120 \mathrm{meV}$. The $4 s \rightarrow 4 p$ ionic transition frequency is approximately $25300 \mathrm{~cm}^{-1}(\lambda \sim 395 \mathrm{~mm})$ and neutral calcium is ionized by absorbing two of the corresponding blue photons. Additional levels present in the calcium atom (Fig. 1) which are also coupled by the laser field 
complicate the minimal model described above. For instance, the $|4 p, \epsilon\rangle \Leftrightarrow|5 s, \epsilon\rangle$ and the two-photon $|4 s, \epsilon\rangle \Leftrightarrow|5 s, \epsilon\rangle$ couplings can be of the same order of magnitude as the $|4 s, \epsilon\rangle \Leftrightarrow|4 p, \epsilon\rangle$ coupling. The presence of additional peaks may be traced to the influence of the fine structure of the $4 p$ ionic state [17]. Likewise, calculations [13] show that neutral resonances such as the $4 s^{2} \rightarrow 4 s 4 p$ transition can also contribute.

The experiment uses a frequency-doubled, regeneratively amplified titanium sapphire laser which produces tunable $(380-405 \mathrm{~nm}), 180 \mathrm{fs}$ pulses. The pulse bandwidth $(\sim 15 \mathrm{meV})$ is less than twice the transform limit and the intensity fluctuations are $\leq 6 \%$. Spectral measurements were made on the fundamental light with a monochromator and an optical multichamel analyzer calibrated with a krypton arc lamp. The spectral resolution was $0.5 \mathrm{~nm}$. The calcium was produced in an $775 \mathrm{~K}$ atomic beam and background contamination was less than $0.01 \%$. Various lenses with $f$-numbers ranging from 7 to 25 focused the light into the atomic beam. The laser's confocal length exceeded the atomic beam's crosssectional length, ensuring a flat intensity distribution in the interaction volume. Electron energy analysis was performed with a time-of-flight spectrometer with $2 \pi$ solid angle collection and an energy resolution of $30 \mathrm{meV}$.

Figure 2 shows the change in the photoelectron energy spectrum (PES) with increasing photon energy (bottom to top) for the low and high intensity limits. In the low intensity perturbative limit [Fig. 2(a)], the spectra reflect 2-photon excitation to an unperturbed $4 \dot{s}$ ion ground state. The electron emission is con-

(a) Low Field

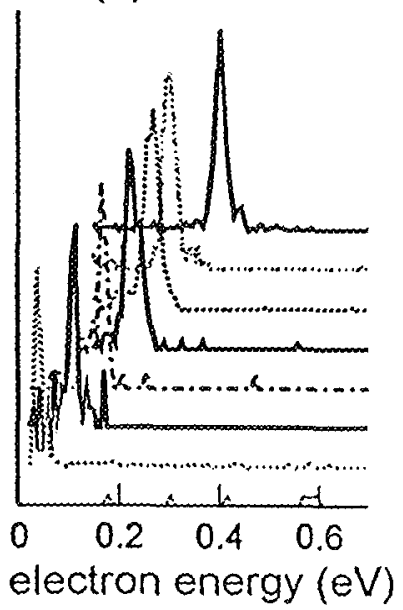

(b) High Field

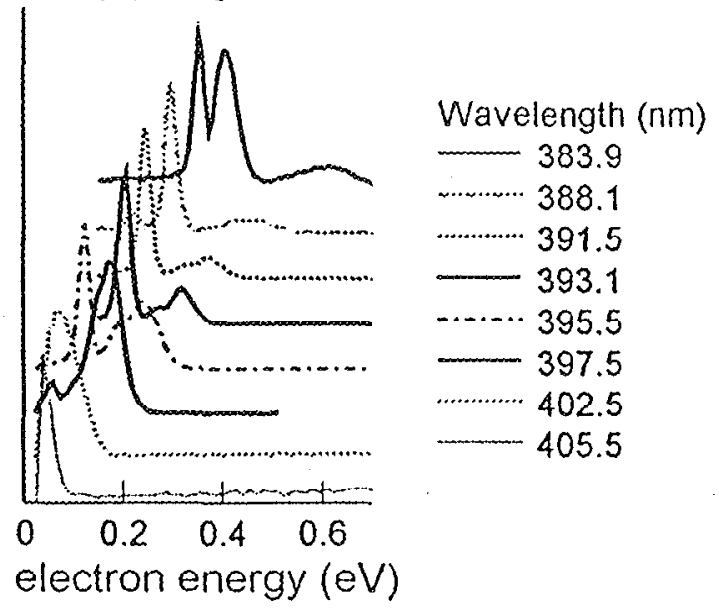

electron energy (eV)

EIGURE 2. PES for calcium at several wavelengths at intensities of (a) 10 and (b) $300 \mathrm{GW} / \mathrm{cm}^{2}$. The solid lines correspond to "on" resonance spectra. 
(a) ON Resonance

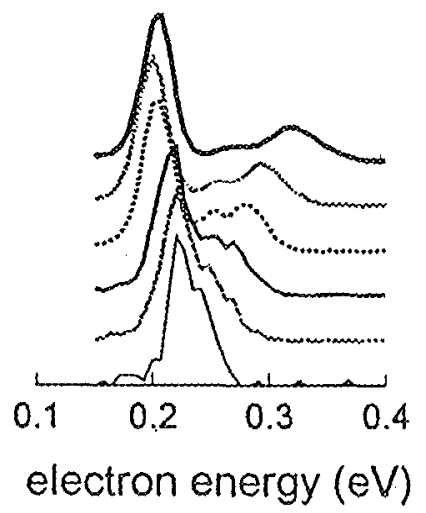

(b) OFF Resonance

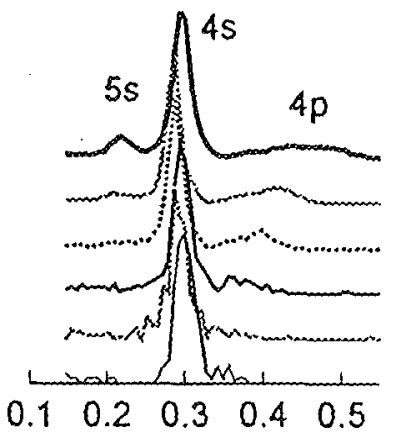

electron energy (eV)

FIGURE 3. Electron spectra (a) "on resonance" $(\lambda=393.5 \mathrm{~mm})$ and "off resonance" $(\lambda=388.1$ $\mathrm{nm})$ for different intensities labeled in fraction of the saturation intensity $\left(300 \mathrm{GW} / \mathrm{cm}^{2}\right)$.

fined in a narrow peak $\left(\sim 30 \mathrm{meV}\right.$ width) centered at $\left(2 E_{\omega}-I P\right)$, where $I P$ is the neutral calcium ionization potential $(6.11 \mathrm{eV})$. The high intensity spectra shown in Fig. 2(b) are taken at the 2-photon saturation intensity $\left(300 \mathrm{GW} / \mathrm{cm}^{2}\right)$ for neutral ionization. Examination of the $(\mathrm{m} / \mathrm{q})$ resolved total yields reveals that the fraction of $\mathrm{Ca}^{2+}$ present at this intensity is negligible $\left(<10^{-3}\right)$. Several new peaks appear for wavelengths shorter than the $4 s \rightarrow 4 p_{1 / 2}$ core resonance $(397 \mathrm{~nm})$ whose relative amplitudes evolve with the laser wavelength. The most prominent features appear at wavelengths near the 1-photon $4 s \rightarrow 4 p_{3 / 2}(393.5$ $\mathrm{nm})$ and 2-photon $4 s \rightarrow 5 s(383.4 \mathrm{~nm})$ core transitions. The same features are reproduced in the ATI peaks e.g. $(2+1)$-photon ionization, with amplitudes which are a few percent of those of the main peak for wavelengths shorter than $400 \mathrm{~nm}$.

Figure 3 shows the intensity dependence of the photoelectron energy spectra at constant wavelength. In Fig. 3(a) the laser is tuned "on resonance" with the ionic $4 \mathrm{~s}_{1 / 2}-4 \mathrm{p}_{3 / 2}$ transition $(393.5 \mathrm{~nm})$ for intensities ranging from about $10^{10}$ to $3 \times 10^{11} \mathrm{~W} / \mathrm{cm}^{2}$. The Rabi splitting for this intensity range varies from 4 to $120 \mathrm{meV}$. At the lowest intensity only one peak emerges at the expected energy for the two-photon ionization, with a small shoulder evident on the high energy side. As the intensity increases, the main feature is red-shifted while the shoulder develops into new structures on the high energy side becoming progressively blueshifted. In fact, the blue shifted structure resolves into a cleav doublet, and the relative amplitude of the two components depends on the intensity. The splitting at saturation is $120 \mathrm{meV}$ and scales as the $\sqrt{I}(\propto E)$, as expected for an "on" resonance scenario. The laser is tuned "off resonance" in Fig. 3(b) B Besides the trivial shift due to the change in photon energy, the intensity dependence of the spectrum is somewhat different: the main peak is basically unshifted, a weak component is increasingly blue-shifted and at the highest intensity, a new feature appears on the red side of the main peak. 
The above experiment demonstrate a qualitative behavior as a function of the wavelength and intensity which is certainly consistent with the predictions of the continuum-continuum Autler-Townes model. Our analysis [17] and the work by several other groups $[12,13,18]$ show that it is the complexity of the atomic structure which produces deviations beyond the minimal model [10]. All calculations rely on an "essential states" approximation and give excellent agreement with the above measurements. However, the experiment clearly illustrates the importance of correlation in a strongly-coupled two electron atom and the ability of an intense laser field to modify continuum structure.

\section{Double Ionization in the Strong Field Limit}

As formalized by Keldysh [7] in 1965, the character of ionization changes with increasing intensity. In weaker fields a bound electron will be promoted into the continuum by the simultaneous absorption of enough photons to increase its energy above its ionization potential. This is called multiphoton ionization (MPI). However, as the laser intensity increases, a completely different node of escape becomes possible. At large distances from the nucleus the electrostatic attraction of the ion core can be overwhelmed by the laser's instantaneous electric field, producing a barrier through which the valence electron can tunnel. In this regime a quasi-static tunneling picture becomes appropriate: the laser field varies so slowly compared to the response time of the electron that the ionization rate becomes simply the cycle-average of the instantaneous dc-tunneling rate. In the language of Keldysh, tumneling ionization (TI) becomes dominant when the ratio of the frequency of the applied field to the tumneling rate becomes less than unity.

The Keldysh theory prediction of the evolution to TI in strong fields has been confirmed by various rigorous theoretical methods [19]. However, experimental access to the tunneling regime has been limited, hampering quantitative comparisons with various strong-field models. The reason for this is simply that for visible laser pulses, even as short as $50 \mathrm{fs}$, jonization depletes the ground state (saturation) before the atom can experience intensities where $\gamma<1$. Consequently, the majority of experimental studies on neutral atoms exposed to intense, short pulse laser fields have been carried out in the MPI or mixed regime $(\gamma>1)$.

Recently a comprehensive understanding of the underlying dynamics of how a tunnel ionized electron leaves the atom has been achieved. The reader is referred to recent review paper for a more complete treatment [20]. This advance has been driven by significant progress in both experimental and theoretical capabilities. Experimentally, the advent of kilohertz repetition rate, high peak power lasers [21] has provided an essential tool necessary to span the entrre intensity range of importance. At the same time numerical solutions of the time-dependent Schroedinger equation have provided accurate and informative views of the excited electron dynamics [22]. The culmination of these is an intuitive model of strong field rescattering $[23,24]$ based on simple quasi-classical notions. Once an 
electron in a strong field has made the transition into the continuum from its initial bound state, its motion is dominated by its interaction with the external laser field. Approximately one-half of an optical cycle after the electron enters the continuum, the field can drive the electron back into the vicinity of the ion core where it can undergo elastic or inelastic scattering, or be recaptured into the initial ground state by emitting a high energy photon (high harmonic generation). The essential physics underlying the production of the observed high energy photons and electrons is contained in these (re)collision events.

In this section, we describe a series of systematic studies of the strong-field ionization of helium and neon atoms in the tumneling regime. It has been shown $[6,25]$ that because of their large binding energies, these two atoms tunnel ionize $(\gamma \sim 0.5)$ near saturation with femtosecond, titanium sapphire pulses. Thus, these atoms form a paradigm for our theoretical and experimental investigation of the subtle consequences produced by the rescattering of a tunneled wave packet with its parent ionic core. Furthermore, these experiments provide unambiguous evidence for double ionization in the tunncling limit, insights into the dynamics and stringent tests for theoretical models.

Let us begin by examining the experimental evidence for double ionization. Figure 4 shows the helium ion yield curves for $160 \mathrm{fsec}, 780 \mathrm{~nm}$ excitation. Each data point (symbols) contains $\geq 60,000$ laser shots. Results from five separate scans with three different spot sizes are plotted. It should be noted that the data spans twelve-orders of magnitude in counting range which is only possible duc to the enhanced utility of high powered, kilohertz repetition rate lasers. The $\mathrm{He}^{+}$yield increases nonperturbatively up to a measured saturation intensity of $8 \times 10^{11} \mathrm{~W} / \mathrm{cm}^{2}$. Beyond this point the yield increases as $\mathrm{I}^{3 / 2}$ consistent with an expanding gaussian focal volume, a purely geometric growth. However, the $\mathrm{He}^{2+}$ curve shows the characteristics of two rate linetics; nonsequential (NS) twoelectron production at low intensities $\left(1.5-8 \times 10^{14} \mathrm{~W} / \mathrm{cm}^{2}\right)$, a saturated regime $\left(0.8-3 \times 10^{15} \mathrm{~W} / \mathrm{cm}^{2}\right)$, sequential production above $3 \mathrm{PW} / \mathrm{cm}^{2}\left(H e^{+} \rightarrow H e^{2+}\right)$ and saturation at $8 \mathrm{PW} / \mathrm{cm}^{2}$. It is this behavior in the $\mathrm{He}^{2+}$ yield which suggests the existence of correlated double ionization.

Further inspection of the data reveals some additional clues into the double ionization mechanism. First, Fig. 4 shows the $\mathrm{He}^{+}$yields calculated by numerically solving the time-dependent Schroedinger equation using a single-active electron (SAE) approximation [23] (solid line) and ac-tunneling (ADK) rates [26] (dashed line). SAE provides the total He ionization rate, including both the multiphoton and tunneling pathways. For the $\mathrm{He}^{+}$yield, both calculations agree with the data at high intensity but the ADK curve falls below the measured yield at low intensity. By contrast, the SAE results are in agreement over the full dynamic range of the experiment showing the multiphoton contribution becomrirg increasingly important below $0.5 \mathrm{PW} / \mathrm{cm}^{2}$. For the $\mathrm{He}^{2+}$ yield, the $\mathrm{SAE}$ and ADK result in overlapping curves (solid line) and agree with the measured yield above $3 \mathrm{PW} / \mathrm{cm}^{2}$. All the $\mathrm{He}^{2+}$ yield at low intensity is beyond any $\mathrm{SAE}$ approximation. Second, note that the $\mathrm{He}^{+}$and $\mathrm{He}^{2+}$, labeled NS, yields follow each other over ten 


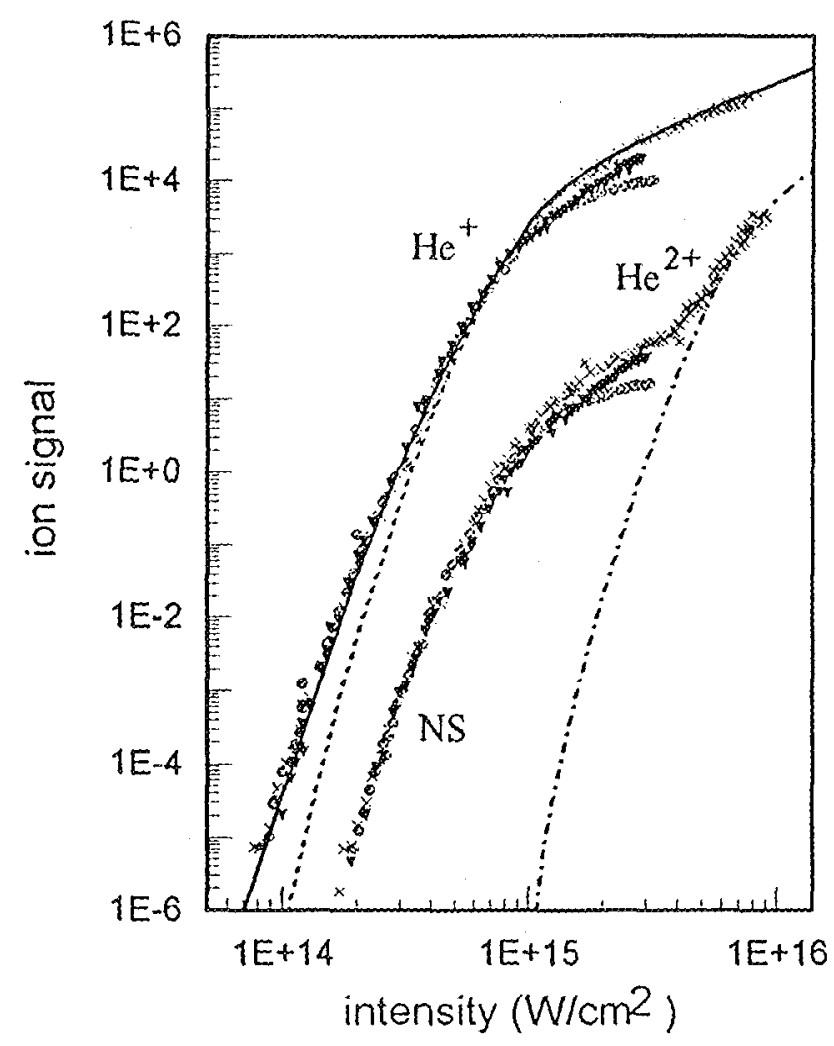

FIGURE 4. Measured He ion yields for linear polarized, $100 \mathrm{fsec}, 780 \mathrm{~mm}$ light. Calculations are shown as solid (SAE) and dashed (ac-tumneling) lines. The dashed-dotted curve on right is the calculated sequential $\mathrm{He}^{2+}$ yield.

orders of magnitude in signal, saturating simultaneously. This behavior, verified by measurements [6], firmly establishes that the NS production is connected with the depletion of ground state neutral helium. Third, the efficient NS ionization is unlikely to be attributable to resonance effects because the helium doubly excited states are well above the first ionization threshold, by over $35 \mathrm{eV}$, so that they cannot be expected to be strongly excited by the optical field. Finally, the NS rate is found to have a much stronger dependence on the ellipticity of the laser field than the sequential process $[27,28]$ and is essential extinguished with circular polarized light.

A sensitive measure of the NS dynamics is provided by plotting the intensity dependence of the $\mathrm{He}^{2+} / \mathrm{He}^{+}$ratio, shown in Fig. 5. To ensure accuracy, the two ions were concurrently collected at a fixed intensity and averaged for at least $10^{6}$ laser shots. Although the ion curves in Fig. 4 show a strong intensity dependence varying by 7 -orders of magnitude between 0.15 and $5.0 \mathrm{PW} / \mathrm{cm}^{2}$, the ratio exhibits a gentle slope of only $I^{1.3}$. The ratio is constant $(0.0020[3])$ from about 5 $\mathrm{PW} / \mathrm{cm}^{2}$ until the sequential production of $\mathrm{He}^{2+}$ becomes significant. The onset of sequential $\mathrm{He}^{2+}$ production is corroborated by the unambiguous appearance 


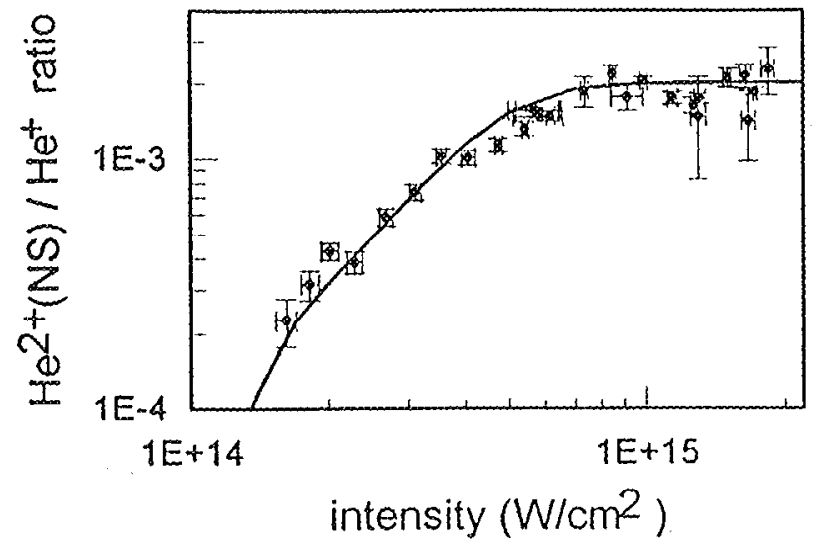

FIGURE 5. Intensity dependence of $\mathrm{He}^{2+}(\mathrm{NS}) / \mathrm{He}^{+}$ratio for $780 \mathrm{~nm}$. Error bars indicate 1 standard deviation. Solid line is calculated; sec text for details.

of a high energy tail in the PES beyond $3 \mathrm{PW} / \mathrm{cm}^{2}[6]$. Assuming that the NS rate is given by the pure ac-tunneling rate times a constant, which is defined by the measured ratio of $\mathrm{He}^{2+} / \mathrm{He}^{+}$at saturation, the dotted NS yield curve in Fig. 4 results. Furthermore, the ratio of this curve to the $\mathrm{SAE} \mathrm{He}{ }^{+}$yield curve produces the solid line in Fig. 5. The striking agreement with the data implies that tunneling is correlated with NS double ionization. We emphasize two differences between tunneling and MPI. First, in tunneling, electrons are emitted in bursts near the maxima in the oscillating electric field while the multiphoton excitation is constant throughout the optical cycle. Second, the multiphoton ionized electrons appear in the continuum near the nucleus whereas tumneling electrons originate at the outer turning point of the instantaneous potential barrier, $6 \sim 10 a_{o}$ from the nucleus. These differences mean that the dynamics of the electrons, after reaching the continuum by these separate pathways, can be significantly different.

We have tested [25] strong-field rescattering using a complete quasi-classical model which incorporates recollision of a field-driven electron with realistic core potentials. We divide the optical cycle into a large number of equal time intervals. The model assumes that at each phase a tunnel ionized wave packet propagates in the combined fields of the laser and the ion core along the classical trajectories. The wave packet is a freely spreading gaussian which is allowed to have only a single return to the core. We can then calculate the differential elastic scattering cross section for comparison to the measured photoelectron distributions. While the e-2e inelastic process, which leads to the production of double ionization, is calculated using a modified Lotz cross section [29] which accounts for beth excitation and ionization. Spatial and temporal averaging is performed for comparison to the experimental measurements.

The calculation yields excellent agreement for the photoelectron energy and angular distributions for both helimm and neon. Figure 6 shows the measured 


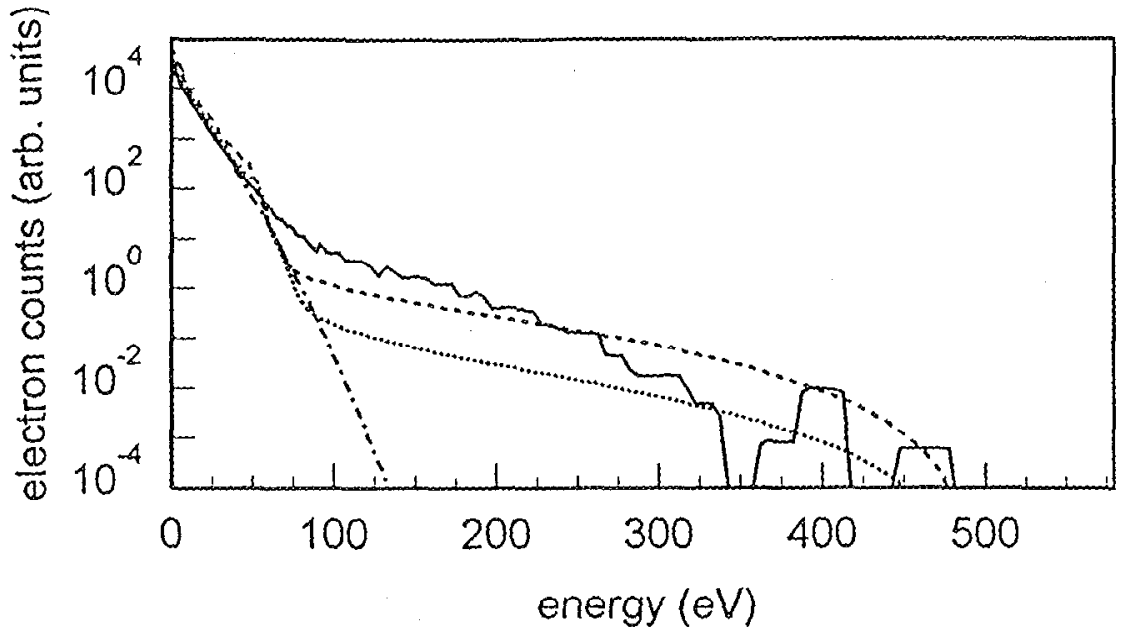

FIGURE 6. Total helium PE energy distribution for $0.78 \mu m$ excitation $8 \times 10^{14} \mathrm{~W} / \mathrm{cm}^{2}$. The experimental and calculated distributions using the complete semi-classical theory presented here correspond to the solid and dashed lines, respectively. The dotted line results from pure Coulomb rescattering and the dashed-dotted is without rescathering. The Keldysh parameter, $\gamma$, equals 0.5 .

(solid line) total PES for helium at $0.8 \mathrm{PW} / \mathrm{cm}^{2}$ compared to three different calculate curves. The dashed-dotted curve is the result calculated in the absence of rescattering and shows the necessity of the additional electron-core interaction for producing high energy electrons. The dashed curve incorporates rescattering with a realistic helium core potential while the dotted curve is pure Coulomb. The importance of the short range physics is exemplified by the better agreement achieved with a realistic potential. Similar agreement is found at different intensities, as well as for neon ionization. Again, we find that the use of a realistic core potential is a necessity.

The complete quasiclassical calculation, described above, can be used to predict the double-to-single ionization ratio produced from e-2e inelastic rescattering. Figure 7 shows the measured (open circles) and calculated (solid line) ratio for helium and the measured (solid circles) and calculated (dashed line) ratio for neon. These results are computed using the same initial conditions and core potentials used to calculate the photoelectron spectrum of Fig.6. A modified "field-free" e-2e Lotz cross-section [29] is used to account for double ionization contributions from both core excitation and direct ionization. In these strong fields, the returning electron needs only to excite one of the remainims electrons of the ion in order to produce the doubly charged ion. Any excited state will be immediately ionized when the oscillating field of the laser reaches its next maximum. Also, it has been shown [30] that the use of field-free cross sections is a reasonable approximation since the slowly varying electric field from the laser 


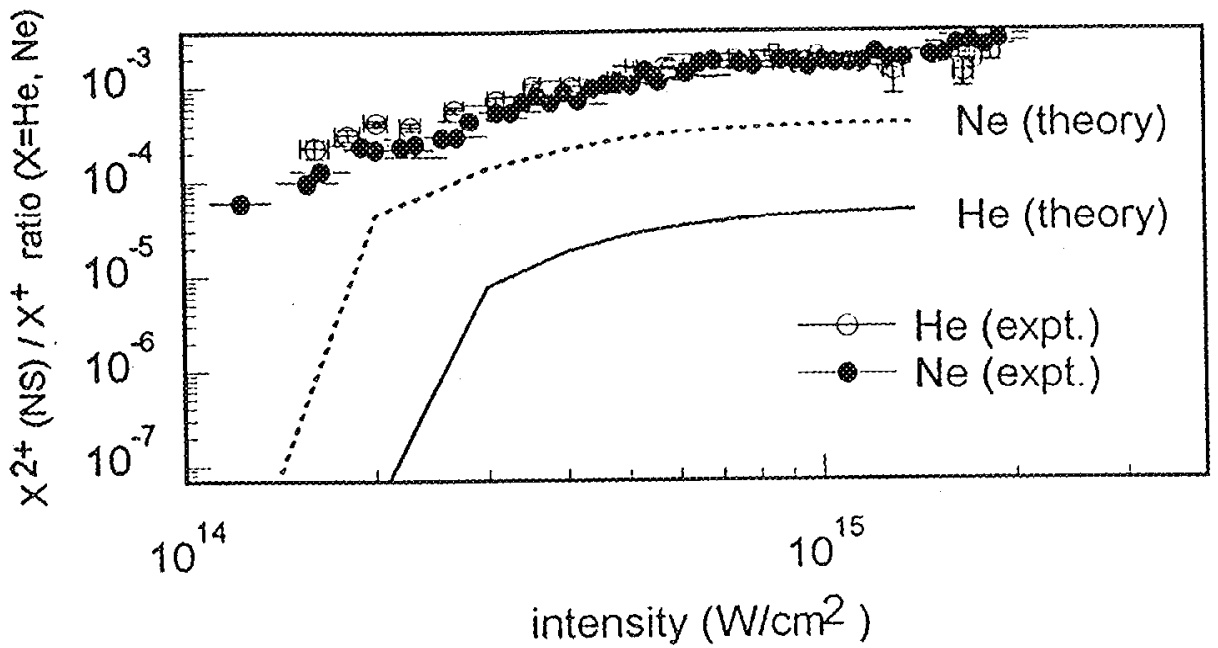

FIGURE 7. Compiled experimental (symbols) and calculated (lines) ratio of nonsequential double ionization to single ionization for helium and neon for $0.78 \mu m$ excitation. The helium and neon calculated curves are given by the solid and dashed lines, respectively.

has a very small effect on the inelastic scattering processes. Clearly, the e-2e rescattering severely underestimates the absolute measured ratio, as well as the shape. The ratio of the experimental to calculated value at saturation is 47 for helium and 5 for neon. The lack of agreement suggests that more than inelastic rescattering is involved in the physics of the nonsecquential ionization.

The disagreement between the rescattering predictions and the experiment goes beyond underestimating the absolute value. As seen in Fig. 7, the experimental data shows a smooth decrease in the ratio with decreasing intensity, whereas the calculations show a sharp and abrupt cutoff. The origin of the cutoff is clear, as the intensity is lowered the electron's return energy clecreases to the point that it can no longer free the second electron. Again, no such behavior is seen in the experiment. Additionally, it is difficult to rationalize in a rescattering picture why the double ionization ratios would be the same for helium and neon considering the order of magnitude difference in the e-2e cross sections. The good agrecment found between the complete quasiclassical calculation and the experimental electron distributions demonstrated the important distinction produced by the atom's short-range potential. Obviously the calculated curves in Fig. 7 refiect the difference in the ionization cross sections, while the experiment does not.

The disagreement between the measured and calculated double ionization ratio could indicate deficiencies in our rescattering model. Certainly our assumptions of the wave packet spread and cross sections which yicld accurate predictions for the electron distributions seen in Fig. 6 support the estimates used in our model. Two other relevant approximations are that the e-2e inclastic cross section is unaffected by the external field and that the wave packet has a maximum of one 
interaction with the core. The consequences of our first assumption was examined using a semiclassical model for helium [30]. It was found that the oscillating field has negligible effect on the collision-induced transition probabilities of the more tightly bound ion core states. This means that using field-free cross sections in model calculations for the intensities that ionize the first electron will yicld reasonably accurate results.

A rescattering calculation by Brabec et al. [31] which treats both electrons classically, examines the influence of higher order rescattering. The main conclusion of this study was that, although there is a small probability for impact ionization of the bound electron during the first return of the free electron, inclusion of additional returns can significantly enhance the efficiency of double ionization by trajectories with low drift velocities. This leads to an overall factor of 30 increase in the total NS yield relative to that obtained by considering only the first return. Brabec et al. attribute this enhancement to the refocusing of the trajectories by the Coulomb field so that later returns produce much higher charge density near the nucleus. They conclude that this refocusing overwhelms the transverse expansion of the TI wave packet while it propagates (most of the time) in the region beyond the effective range of the ion core potential.

We can test the importance of refocusing for a real, quantum tunnel ionized (TI) wave packet using the SAE approximation to calculate the strength of the generated high harmonics as a function of time after the wave packet is created. Since the harmonics are produced by transitions back to the ground state, this is an ideal probe of the density distribution of rescattering electrons near the nucleus. We use a constant intensity pulse to produce a TI wave packet during the - first half cycle. At this point, the time-dependent wave function is orthogonalized to the ground state and the subsequent evolution represents only that of the excited state component of the total wave function. As this TI wave packet is driven back and forth across the the ion core, we can Fourier transform (FT) the dipole matrix element between the wave packet and the ground state. We can consider the spectra generated by different "retums" by restricting the time interval in the FT. We find that the emission rate during the first return is at least a factor of ten stronger than that from the next two cycles, with later returns falling by more orders of magnitude. We must conclude that the Coulomb focusing is not sufficient to explain the substantial enhancement Brabec et al. find in their trajectory calculations. A more likely explanation for their result is that when the TI electron first returns, it can transfer a small amount of energy to the bound electron, becoming trapped in a low-lying "doubly excited" state. These states, which cannot exist in the quantum system, are allowed classically because the density of states is continuous. The captured electron requires more collisions before it can re-escape. This will produce a very large enhancement of the NS ionization yield that would be completely absent in the real, quantum system.

A number of more elaborate two-electron quantum calculations [32-34] have been reported to explain the helim measurements described above. However, treatment of these results are beyond the scope of this paper and the reader is 
referenced to the appropriate papers. Each of these calculations give reasonable agreement with the helium doulje ionization for linear polarized light presented above. In fact, the conclusion of Ref. [34] is that rescattering mechanism dominates. However, we feel that considering the more global experimental evidence and some of the model approximations, no convincing theoretical demonstration of any explicit mechanism of strong field double ionization currently exists. Over twenty years has passed since the first observation of Suran and Zapesochnyi [3], yet the question of how electron correlation influences strong-field double ionization remains largely unanswered and remains a significant challenge for future investigations.

\section{ACKNOWLEDGMENTS}

This research was carried out in part at Brookhaven National Laboratory under contract No. DE-AC02-98CH10886 with the U.S. Department of Energy and supported by its Division of Chemical Sciences, Office of Basic Energy Sciences, and in part under the auspices of the U. S. Department of Energy at the Lawrence Livermore National Laboratory under contract No. W-7405-ENG-48. L. F. D. and P. A. acknowledge travel support from NATO under Contract No. SA.5-205(RG910678).

\section{REFERENCES}

1 Fano, U. and Rau, A.R.P., Atomic Collisions and Spectra, New York: Academic Press, 1986.

${ }^{2}$ Chang, T.N., Many Body Theory of Atomic Structure and Photoionization, Singapore: World Scientific, 1993.

3 Suran, V.V. and Zapesochnyi, I.P., Sov. Tech. Phys. Lett. 1, 420 (1975).

${ }^{4}$ Lambropoulos, P., Maragakis, P. and Zhang, J., Phys. Repts. (1998) in press and references therein.

5 Walker, B., Kaluža, M., Sheehy, B., Agostini, P. and DiMauro, L.F., Phys. Rev. Lett. $75,633(1995)$.

6 Walker, B., Sheehy, B., DiMauro, L.F., Agostini, P., Schafer, K.J. and Kulander, K.C., Phys. Rev. Lett. 73, 1227 (1994).

7 Keldysh, L.V., Zh. Eksp. Teor. Fiz. 47, 1945 (1964).

${ }^{8}$ Autler, S.L. and Townes, C.H., Phys. Rev. 100, 703 (1955).

9 Mollow, B.R., Phys. Rev. A. 5, 2217 (1972).

10 Grobe, R. and Eberly, J.H., Phys. Rev. A 48, 623 (1993).

11 Robicheaux, F., Phys. Rev. A 47, 1391 (1993).

12 Grobe, R. and Llaan, S.L., J. Phys. B 27, L735 (1994).

13 Hanson, L.G., Zhang, J. and Lambropoulos, P., Europhys. Lett. 30,81 (1995).

14 Knight, P.L., J. Phys. B 11, L511 (1978).

15 Hanson, L.G. and Lambropoulos, P., Phys. Rev. Lett. 74, 5009 (1995). 
16 Allen, L. and Eberly, J.H., Optical Resonance and Two-level Atoms, New York: John Wiley \& Sons, 1975.

17 Walker, B., Sheehy, B., Kaluža, M., DiMauro, L.F., Trahin, M. and Agostini, P., in Super Interse Laser Atom Physics IV: NATO ASI Series, Dordrecht: Kluwer Publishing, 1996, pp. 295-304.

18 Haan, S.L., Bolt, M., Nymeyer, H. and Grobe, R., Phys. Rev. A 51, 4640 (1995).

19 Kulander, K.C. and Schafer, K.J., Multiphoton Processes, Singapore: World Scientific, 1993, pp 391-409.

20 For a review, see DiMauro, L.F. and Agostini, P., in Advances in Atomic, Molecular, and Optical Physics 35, San Diego: Academic Press, 1995, pp 79-120.

21 Saeed, M., DiMauro, L.F. and Tornegard, S., Laser Focus World 27, 57 (1991).

22 For a review of time-dependent methods, see Kulander, K.C., Schafer, K.J. and Krause, J.L., in Atoms in Intense Radiation Fields, New York: Academic Press, 1992, pp. 247-300

23 Schafer, K.J., Yang, B., DiMauro, L.F. and Kulander, K.C., Phys. Rev. Lett. 70, 1599 (1993).

24 Corkum, P.B., Phys. Rev. Lett. 71, 1994 (1993).

25 Walker, B., Sheehy, B., Kulander, K.C. and DiMauro, L.F., Phys. Rev. Lett. 77, 5031 (1996).

26 Ammosov, M.V., Delone, N.B. and Krainov, V.Y., Soviet Phys. JEPT 64, 1191 (1986).

27 Walker, B., Mevel, E., Yang, B., Berger, P., Chambaret, J.P., Antonetti, A., DiMauro, L.F. and Agostini, P.A., Phys. Rev. A 48, R.894 (1993).

28 Dietrich, P., Burnett, N.H., Ivanov, M.V. and Corkum, P.B., Phys. Rev. A 50, R3585 (1994).

29 Lotz, W., Z. Phys. 216, 241 (1968).

30 Kulander, K.C., Cooper, J. and Schafer, K.J., Phys. Rev. A A51, 561 (1995).

31 Brabec, T., Ivanov, M.V. and Corkum, P.B., Phys. Rev. A 54, R2551 (1996).

32 Becker, A. and Faisal, F.H.M., J. Phys.B 29, L197 (1996).

33 Faisal, F.H.M. and Becker, A., Laser Phys. 7, 684 (1997).

${ }^{34}$ Watson, J.B., Sanpera, A., Lappas, D.G., Kuight, P.L. and Burnett, K., Phys. Rev. Lett. 78, 1884 (1997). 